\title{
One lung ventilation: double-lumen tube with vs. without carinal hook
}

\author{
IZTOK POTOČNIK, LEA ANDJELKOVIĆ, JASMINA MARKOVIČ-BOŽIČ
}

Clinical Department of Anaesthesiology and Surgical Intensive Therapy, University Medical Centre Ljubljana, Slovenia

\author{
Correspondence: \\ Iztok Potočnik \\ Clinical Department of Anaesthesiology and Surgical Intensive Therapy \\ University Medical Centre Ljubljana \\ Zaloška 2, Ljubljana, Slovenia \\ Phone: 0038640397119 \\ E-mail: iztok.potocnik@kclj.si
}

\section{ABSTRACT}

Background. One lung ventilation (OLV) has become a standard procedure for the vast majority of interventions in pulmonary surgery. The most commonly used are left sided double-lumen tubes (DLTs) which are placed into the left main bronchus and the right or left lung can be isolated.

The aim of our study was to compare DLTs with and without a hook.

Materials and methods. Fifty-four patients undergoing lung resection were included in the randomized, controlled, single-blinded study. Recruited patients were randomly allocated to each group (hook/without hook). Demographic data, procedural data, type of tube used, and difficult intubation criteria were recorded. Complications, according to intubation and position of the tube, were also recorded. After the operation, we aske patients about a sore throat, hoarseness, haemoptysis and their satisfaction with the procedure.

Results. Baseline characteristics were well balanced between groups. Time to place DLT was shorter in the group without a hook $(47.7 \pm 45.5$ vs $15.8 \pm 15.1 \mathrm{~s} ; \mathrm{P}=0.01)$. The incidence of adequate positioning at the first attempt was higher in the group with a hook and the repositioning rate was higher in the group without a hook (22.22 vs 59.25\%; $\mathrm{P}=0.004)$. Patients in both groups suffered similar incidences of hoarseness, sore throat or postoperative haemoptysis (5/5/1 vs $3 / 3 / 0 ; \mathrm{P}=0.44)$. Patient satisfaction was higher in the group without a hook (31.85\% vs $34.81 \% ; 0.03$ ).

Conclusion. The study showed the advantage of DLTs without a hook in comparison with DLTs with a hook. In our institution we decided to use DLTs without a hook, with fiberoptic control.

Key words: double-lumen tube, carinal hook, pulmonary surgery

\section{INTRODUCTION}

One lung ventilation (OLV) has become a standard procedure for the vast majority of interventions in pulmonary surgery. It is used in both techniques: thoracotomy and videothoracoscopy (VATS). OLV can be provided by a double lumen tube (DLT) or bronchial blocker. There are advantages and disadvantages of both techniques, but a DLT is recommended more often because it allows total emptying of the operated lung. Air and secretions can be aspirated through the wide lumen of the tube during surgery. (1-3) The first DLT was used in 1949, when Carlens invented the left sided DLT with the hook. (4) The hook is placed on the carina to prevent displacement of the tube. DLTs with a hook also help blind placement on the carina. (4) Nowadays there are many variations of DLTs and bronchial blockers which differ according to shape and material. $(5,6)$ There are also disposable polyvinylchloride Robertshaw DLTs with or without a hook. DLTs without a hook are more gentle and easier to place in the left main bronchus. (7) After insertion of the left tube without a hook, bronchoscopy is recommended to check the position of the tube. (8-11) There is no consensus on the best technique for lung isolation for thoracic surgery. (12) The most commonly used are left sided DLTs which are placed into the left main bronchus and the right or left lung can be isolated. Descriptions of some severe complications (injury of the bronchial tree) after insertion of a hooked tube can be found in the literature. (13) We have published such an example, based on our earlier experience. (14) The use of doublelumen tubes with a hook is widespread in Europe and quite unknown in the United States. Each anaesthesiologist decides individually which kind of DLT to use as the literature is poor, containing only a few case reports. There is only one study where they have compared both techniques but no difference was found. (15) That is why we decided to study which technique is better so we can include it in our standard operative procedure.

\section{MATERIALS AND METHODS}

This is a single-centre, controlled, randomised, single-blinded study. It was approved by the independent Slovenian Ethical Committee. The study protocol was registered under Clinical Trial number NCT02857504. Each patient received detailed oral and written information and gave final consent before surgery. We included in the study patients booked for planned thoracotomy or VATS surgical technique, with American Society of Anesthesiologists (ASA) physical status $1-3$, aged 18 years or older, requiring left sided DLT for one lung ventilation. We excluded from the study patients with ASA $>3$, severe heart disease (NYHA $>3$ ), severe pulmonary obstructive disease (FEV1<40\%), neurologic or psychiatric disorders, patients with Mallampati or Cormack-Lehane score more than 3, criterion for expected difficult intubation and with risk factors for pulmonary aspiration. Recruited patients were randomly allocated 1:1 to each group with a computer programme (hook group, no hook group). The main criteria for difficult intubation (Mallampati score, neck movement and opening of the month) were determined before the operation. We recorded demographic data, all procedural data, the type of tube used and its size and Cormack-Lehane score. We monitored complications related to intubation and positioning of the tube. After the operation we asked patients 
about any pain in the throat, hoarseness and how satisfied they were with the procedure. Intraoperative and postoperative management were the same in both groups according to the Standard operative protocol for this kind of surgery at the University Clinical Centre Ljubljana in Slovenia. (16) Patients received midazolam $7.5 \mathrm{mg}$ orally one hour before the procedure. In the operating room, standard monitoring was performed and peripheral and arterial cannulas were inserted. (16) C-Mac videolaringoscopy (Storz) was used for intubation. Patients from the "hook group" were intubated with the hook DLT (Bronchocath; Malinckrodt, Athlone, Ireland)) and those from the "no hook group" were intubated with the DLT without a hook (Broncho-cath; Malinckrodt, Athlone, Ireland). For intubation, propofol 1-1.5 mg/kg TT, fentanyl $20 \mathrm{mcg} / \mathrm{kg} / \mathrm{TT}$ and rocuronium $0.6 \mathrm{mg} / \mathrm{kg}$ TT were used. After administration of the drugs, we waited 2 minutes for the muscle relaxant to start working properly. We inserted the laryngoscope and evaluated the Cormack-Lechan score. The time needed for tube insertion (time from seeing the vocal cords to final position of the tube-without bronchoscopy) was measured. The tube without the hook was inserted using the following technique: after the bronchial cuff passed the vocal cords, the stylet was removed and the tube was rotated $90^{\circ}$ towards the left. The tube with the hook (after passing the bronchial cuff through the vocal cords) was rotated 180 degrees to the left and the stylet removed. When the hook passed the vocal cords, the tube was rotated 90 degrees back to the right and pushed into the bronchus. The following formula was used to determine the right depth (height $(\mathrm{cm}) / 10+12$ $(\mathrm{cm}))$ of the tube without the hook. The tube with the hook was inserted into the bronchus so that the hook was placed on the carina. The position of the tube was confirmed on auscultation. If this was unclear, a fiberoptic bronchoscope was used. After placing the tube in the right position, we started with ventilation. After positioning the patient in the side position, we checked the tube position one again with the fiberoptic bronchoscope. The lumen of the DLT in the nondependent lung was clamped and its port was opened. Tracheal extubation was performed in the operating room. An investigator not involved in the study and blinded to group allocation of the patient, asked the patient about any postoperative complications and satisfaction with the procedure. The primary outcome of the study was the time, measured using a stopwatch, required to position the tube correctly. The secondary outcome was the incidence of correct positioning of the tube at the first attempt. Two hours after the operation, when the patient was completely awake, we evaluated the following complications: hoarseness, sore throat and haemoptysis. We asked the patient also about his/her satisfaction with the procedure.

\section{STATISTICAL ANALYSIS}

Data were analysed using SPSS 13.0 software package. The two-tailed t-test with unequal variances or the Chi-square test were used to test the differences in demographic data, surgical procedure, time to intubation, adequate position, postoperative complications and patient satisfaction. The means of continuous variables are presented, and categorical data are summarized as counts. A p-value of less than 0.05 was considered statistically significant.

Table 1. Baseline demographics and surgical procedure

\begin{tabular}{llll}
\hline & hook & without hook & $\mathrm{p}$ \\
\hline Number & 27 & 27 & $/$ \\
\hline Age (years) & $61 \pm 14.6$ & $53 \pm 17.5$ & 0.07 \\
\hline Weight $(\mathrm{kg})$ & $74,7 \pm 16.5$ & $74.2 \pm 15.4$ & 0.91 \\
\hline Height $(\mathrm{cm})$ & $172.4 \pm 9.3$ & $169.5 \pm 7.3$ & 0.21 \\
\hline Sex $($ male/female) & $12 / 15$ & $12 / 15$ & 0.61 \\
\hline ASA $(1 / 2 / 3)$ & $3 / 7 / 17$ & $5 / 6 / 16$ & 0.74 \\
\hline Mallampati $(1 / 2 / 3 / 4)$ & $8 / 15 / 4 / 0$ & $9 / 13 / 4 / 0$ & 0.75 \\
\hline Cormack $(1 / 2 / 3 / 4)$ & $21 / 4 / 1 / 1$ & $13 / 8 / 6 / 0$ & 0.05 \\
\hline Tumour/other pathology & $18 / 9$ & $15 / 12$ & 0.29 \\
\hline DLT size $(37 / 39 / 41)$ & $9 / 15 / 3$ & $10 / 13 / 4$ & 0.16 \\
\hline VATS/thoracotomy & $24 / 3$ & $21 / 6$ & 0.23 \\
\hline
\end{tabular}

ASA, American Society of Anaesthesiology; DLT, double-lumen tube; VATS, videothoracoscopy. The results are expressed as mean $\pm \mathrm{SD}$ or number of patients $\mathrm{P}<0,05$ : result is statistically significant

Table 2. Time to intubation, adequate position, postoperative complications and patient satisfaction

\begin{tabular}{llll}
\hline & hook & without hook & $\mathrm{p}$ \\
\hline Time to intubate (s) & $47.7 \pm 45.5$ & $15.8 \pm 15.1$ & $0.01^{*}$ \\
\hline $\begin{array}{l}\text { Adequate position (\%) } \\
\text { Complications (no/hoarse- }\end{array}$ & 22.22 & 59.25 & $0.004^{*}$ \\
$\begin{array}{l}\text { ness/sore throat/haemop- } \\
\text { tysis) }\end{array}$ & & $21 / 3 / 3 / 0$ & 0.44 \\
\hline \begin{tabular}{l} 
Satisfaction (1-10) \\
\hline
\end{tabular} & $8.6 \pm 1.6$ & $9.4 \pm 1.1$ & $0.03^{*}$ \\
\hline
\end{tabular}

The results are expressed as mean \pm SD or number of patients

$\mathrm{P}<0,05$ : statistically significant result 
and patient satisfaction were better in the group without the hook. The complication rate was not significantly different between both groups. Our study can be compared with a recent, large study by Dumas-Nizard et al. (15) They compared intubation using DLTs with a hook and those without a hook. Unlike us, their study showed that the use of DLTs with or without a hook gave similar results especially for the primary outcome - the time to obtain correct placement. Our study showed that adequate positioning is achieved significantly quicker in the group without a hook. Secondary outcomes in the Dumas-Nizard study were similar in both groups, particularly lung collapse, postoperative sore throat and airway complications. (15) In our study, the postoperative complication rate was higher in the group with a hook, but the difference was not statistically significant. All other published studies had compared bronchial blockers and DLTs. Mourisse et al. published a large study with 100 patients. (17) Placement of a DLT was unsuccessful twice. The incidence of initial malpositioning was high and comparable between EZ-blockers (EZBs) (37 of 50) and DLTs (42 of 49) $(\mathrm{P}=0.212)$. Placing single-lumen tubes and EZBs took more time but was rated easier. Quality of lung deflation was comparable. Fewer patients in the EZB group complained of a sore throat at Day 1. There was a higher incidence of tracheal haematoma,redness and bronchial haematoma in the DLT group. They concluded that the EZB is an efficient and effective device for one-lung ventilation and causes less injury and sore throat than a DLT. Similar to Dumas-Nizard et al. (15), we decided to use »time to obtain correct placement « as the primary outcome measure. Time is simple to measure and it relates to the number of adequate tube placements. (15) It can also be compared between different studies, if the definitions are the same. Like Dumas-Nizard et al. (15) and Mourisse et al. (17), we did not find a significant difference in postoperative complications (hoarseness, sore throat, and haemoptysis). In our study we also measured patient satisfaction, which was better in the group without a hook. We can explain this result with the lower rate of postoperative minor complications in the group without a hook. The main limitation of our study is the relatively small number of patients involved in the study. We also did not perform a postoperative fiberoptic examination, because in previous studies numerous patients refused it. We also concluded that the examination is very stressful for the patient and hence avoided it in the study. Moreover, we did not follow up patients after they returned to the surgical ward and so we do not have the results of long-term outcome. In conclusion, our study showed some advantages of DLTs without a hook in comparison to DLTs with a hook. In our institution we decided to use DLTs without a hook, with fiberoptic control.

Trial registration: ClinicalTrials.gov NCT02857504

\section{REFERENCES}

1. Clayton-Smith A, Bennett K, Alston RP, Adams G, Brown G, Hawthorne T, et al. A Comparison of the Efficacy and Adverse Effects of Double-Lumen Endobronchial Tubes and Bronchial Blockers in Thoracic Surgery: A Systematic Review and Meta-analysis of Randomized Controlled Trials. J Cardiothorac Vasc Anesth 2015;29:955-66.

2. Campos JH. Lung isolation techniques. Anesthesiol Clin North America 2001 Sept; 19(3):455-74.

3. Pedoto A. How to choose the double-lumen tube size and side: the eternal debate. Anesthesiol Clin 2012 Dec; 30(4):671-81.

4. Carlens E. A new flexible double-lumen catheter for bronchospirometry. J Thorac Surg 1949;18:742-6.

5. Bauer C, Winter C, Hentz JG, Ducrocq X, Steib A, Dupeyron JP. Bronchial blocker compared to double lumen tubes for one- lung ventilation during thoracoscopy. Acta Anaesthesiol Scandi 2001;45:250-4.

6. Campos JH, Hallam EA, Van Natta T, Kernstine KH. Devices for lung isolation used by anesthesiologists with limited experience: comparison of double-lumen endotracheal tube, Univent torque blocker, and Arndt wire guided endotracheal blocker. Anesthesiology 2006;104:261-6.

7. Oberhofer D, Majerić-Kogler V. Comparison between the rubber Carlens tube and the polyvinylchloride Robertshaw tube for endobronchial intubation. Lijec vjesn 1999 Nov-Dec;121(11-12):345-51.

8. Klein U, Karzai W, Bloos F, Wohlfarth M, Gottschall R, Fritz H, et al. Role of fiberoptic bronchoscopy in conjunction with the use of double-lumen tubes for thoracic anesthesia: a prospective study. Anesthesiology 1998 Feb;88(2):346-50.

9. Slinger PD. Fiberoptic bronchoscopic positioning of double-lumen tubes. J Cardiothorac Anesth 1989 Aug;3(4):486-96.

10. Brodsky JB. Fiberoptic bronchoscopy need not be a routine part of double-lumen tube placement. Curr Opin Anaesthesiol 2004 Feb;17(1):7-11.

11. Cohen E. Double-lumen tube position should be confirmed by fiberoptic bronchoscopy. Curr Opin Anaesthesiol 2004 Feb;17(1):1-6.

12. Narayanaswamy M, McRae K, Slinger P, Dugas G, Kanellakos GW, Roscoe A, Lacroix M. Choosing a lung isolation device for thoracic surgery: a randomized trial of three bronchial blockers versus double-lumen tubes. Anesth Analg 2009 Apr;108(4):1097-101

13. Fitzmaurice BG, Brodsky JB. Airway rupture from double lumen tubes. J Cardiothoracic Vasc Anesth 1999;13:322-9.

14. Potocnik I, Kupsch A, Novak-Jankovic V. Iatrogenic rupture of the trachea caused by double Lumen tube intubation. Cent Eur J Med 2010;5:737.

15. Dumans-Nizard V, Parquin JF, Moyer JD, Dreyfus JF, Fischler M, Le Guen M. Left double-lumen tube with or without a carinal hook. A randomised controlled trial. Eur J Anaesthesiol 2015;32:418-24.

16. Novak-Jankovic V. Standardni operativni postopki (SOP) v anesteziologiji, reanimatologiji in perioperativni intenzivni medicini. Ljubljana: Univerzitetni klinični center, Klinični oddelek za anesteziologijo in intenzivno terapijo operativnih strok, Kirurška klinika, 2009.

17. Mourisee J, Liesveld J, Verhagen A, Van Rooij G, Van der Heide S, Schuurbiers-Siebers O, Van der Heijden E. Efficiency, efficassy, and safety of EZ-blocker compared with left-sided double-lumen tube for one-lung ventilation. Anesthesiology 2013;118:550-61. 